\title{
On-gold recombinase polymerase primer elongation for the electrochemical detection of bacterial genome: mechanism insights and influencing factors
}

\author{
Raquel Sánchez-Salcedo[a], Rebeca Miranda-Castro[a], Noemí de-los-Santos-Álvarez ${ }^{[a]}$ and María \\ Jesús Lobo-Castañón*[a] \\ Published: ChemElectroChem 2019, 6, 793-800 (DOI: 10.1002/celc.201801208)
}

\begin{abstract}
The detection of bacterial pathogens may be accomplished through their genome material. With that aim, current methods usually rely on PCR amplifications, which are timeconsuming and require specialist equipment. Here, we optimized an on-chip isothermal recombinase polymerase amplification (RPA) with chronoamperometric detection, which could be run with simple equipment. A short thiol-modified oligonucleotide, complementary to a fragment of bipA gene of Salmonella spp., is chemisorbed onto the gold chip surface together with $p$-aminothiophenol as a blocking agent. Remarkably, we demonstrate that in the presence of Salmonella genome, this oligonucleotide, acting as a primer in RPA, is directly elongated on the surface, copying the genome and giving rise to an on surface enzyme-tagged dsDNA. After $30 \mathrm{~min}$ of isothermal amplification, the chronoamperometric signal, related to one of the products of the enzyme reaction, increased with the amount of Salmonella genome, which allowed the detection of $10^{5}$ copies. The results of this study may be of general utility in the design of sensors for detecting other bacteria.
\end{abstract}

\section{Introduction}

Methods for isothermal DNA amplification have gained great popularity because of their potential for on-site DNA analysis, thus becoming the alternative choice to the thermal cyclingbased PCR out of the well-equipped laboratories. Among the various isothermal technologies described so far, ${ }^{[1]}$ Recombinase Polymerase Amplification (RPA) has received particular attention due to its simplicity, flexibility, and speed. ${ }^{[2]}$

The mechanism of RPA technology is inspired by the homologous recombination occurring in cells to repair the mismatch of non-complementary base pairs as well as doublestrand breaks in the DNA helix. The keystone of this mechanism is the RecA protein, a DNA-dependent ATPase or recombinase, responsible for the exchange between two DNA strands with similar or identical sequence. The process is as follows: RecA protein associates with a single-stranded DNA (ssDNA) molecule resulting in a nucleoprotein filament that searches for

[a] M. Sc. R. Sánchez-Salcedo, Dr. R. Miranda-Castro,

Dr. N. de-los-Santos-Álvarez, Prof. M.J. Lobo-Castañón

Departamento de Química Física y Analítica

Universidad de Oviedo

Julián Clavería 8. 33006 Oviedo (Spain)

E-mail:mjlc@uniovi.es

Supporting information for this article is given via a link at the end of the document. sequence similarity along a double-stranded DNA (dsDNA) molecule. In an organism, that is in vivo, the recombinase has to scan all genomic DNA. Once sequence homologies are located in dsDNA, the nucleoprotein filament invades the duplex and binds to the complementary strand of the dsDNA, displacing the other strand (the one with the same sequence as the initial ssDNA) ${ }^{[3]}$ This mechanism, which occurs naturally in all living organisms involving RecA-like proteins, was used by Piepenburg et al. ${ }^{[4]}$ to develop an in vitro isothermal DNA amplification. Since 2010, this amplification system is patented by the same researchers ${ }^{[5]}$ and marketed by a single company, i.e. Twist $\mathrm{Dx}^{\mathrm{TM}}$. However, this has not represented a stumbling block for its wide-spread application, as is shown by about 260 peer-reviewed publications found through a search in the database WOS (Web of Science) using as key term "Recombinase Polymerase Amplification, RPA", with 185 of them just in the last 3 years.

Like PCR, RPA requires two different primers that match the 5 '-ends of the target template. Traditionally, these oligonucleotides are, along with the rest of the reagents, free in solution and they are extended to create a new template strand resulting in an exponential amplification. However, unlike PCR where quantification is based on the number of thermal cycles necessary for amplification to be reliably detected, for isothermal amplifications, such as RPA, quantification relies on the time required to exceed an established threshold signal. The ability of RPA to proceed optimally at $37^{\circ} \mathrm{C}$ throughout the entire reaction, therefore with no need for a previous step at $95{ }^{\circ} \mathrm{C}$ to separate the dsDNA, has favored the development of analysis devices with non-complex thermal systems. Accordingly, since the invention of RPA different formats have been reported, which combined the isothermal amplification with the visual detection in lateral flow strips ${ }^{[6]}$, optical detection on a DVD format ${ }^{[7]}$, or electrochemical detection on screen-printed electrodes ${ }^{[8]}$ to measure the amount of RPA-amplified double-stranded DNA in solution. The latter transduction approach, i.e. electrochemicalbased methodologies, has found widespread interest for the detection of pathogens. ${ }^{[9]}$

As a step forward for sensors development, solid-phase RPA methods have been developed by immobilization of one or two primers on a solid support, while the other components are in solution. Bearing in mind that DNA polymerases incorporate free nucleotides to the 3'-end of a DNA strand, primers should be anchored at the 5 '-end to make their elongation possible. Moreover, considering that RPA operation temperature is constant and low, conventional surface chemistry can be successfully applied. The on-surface RPA strategy allows 
integrating DNA amplification, hybridization and detection on the same platform, thus contributing to reduce the analysis time and the likelihood of contamination, as well as paving the road to effective on-site testing. Furthermore, this strategy favors the detection of multiple targets in the same reaction (not parallelized assays but real multiplex analysis), since the surface-confinement of primers leads to space-resolved detection. ${ }^{[10]}$ However, the amplification yield is lower than in solution mainly due to the steric hindrance of the solid support that negatively impacts the enzyme efficiency. To alleviate this issue, the incorporation of a poly $\mathrm{T}$ spacer in the grafted primer is highly recommended. ${ }^{[11]}$

Different approaches have been evaluated to implement solid-phase RPA. One possibility entails the attachment of both primers on the surface. The elongation of the primers gives rise to new templates anchored by one end, while the second end is free and bends over, seeking for its complementary immobilized primer, which is subsequently extended to form a nucleic acid bridge, hence the name "bridge amplification". By including digoxigenin-dUTPs in the RPA mix, it is possible to obtain enzyme-labeled amplicons that were indirectly quantified through the detection of a colored enzyme product. ${ }^{[12]}$ Another less challenging approach involves the immobilization of one primer besides incorporating forward and reverse primers in the solution. This way, amplification process takes place first in the solution leading to a shortened dsDNA that serves as the template for surface amplification. If the attached primer is specific for a region located within the liquid-phase formed amplicon (heminested amplification) enhanced selectivity is then achieved. ${ }^{[13]}$ The use of a stoichiometric ratio of both primers in solution could increase the chances of getting unspecific byproducts like primer dimmers; for this reason, asymmetric conditions, that is, low amount of the immobilized primer with respect to the other one, tend to be added to the reaction mix. ${ }^{[14]}$ As alternative, an even simpler configuration arises from maximizing that asymmetry by anchoring one of the primers and incorporating the other primer in solution. ${ }^{[15-16]}$ However, some mechanistic aspects remain obscure. For example, it is not clear whether amplification on the surface takes place or, on the contrary, the anchored primer simply traps the amplified products in solution. In addition, the extent to which the composition of the RPA reagents affects the on-surface amplification efficiency has not been established due to the rigid format of the RPA kit commercially supplied up to very recently.

In this work, we study the mechanism underlying the solidphase RPA amplification by electrochemical detection as well as some key parameters affecting its efficiency, using the recently marketed liquid form of RPA kit with a more flexible set of reagents. As a proof of concept, Salmonella genome has been used as a target dsDNA. Using one of the RPA primers chemisorbed onto a gold surface, the bacteria genome is copied by elongation of the attached primer and electrochemically detected through the tagged fluorescein incorporated to the dsDNA during amplification. On-surface amplification is optimized, which allowed the quantification of $10^{5}$ genome units in $30 \mathrm{~min}$.

\section{Results and Discussion}

Many have been the scientific publications reporting on different DNA-based methods for the detection of Salmonella, also using RPA technology. ${ }^{[7,14 a, 17]}$ Virtually all these assays have been implemented by targeting functional genes implicated in infectivity and virulence of different species of Salmonella, specifically invA (invasion A) gene. Nevertheless, these genes are prone to variability due to silent mutations in their codons, which means that sequences are not conserved between species, leading to false negative results. Alternatively, Calvó et al. have demonstrated via a PCR-based assay the reliability of the regulatory (therefore, highly conserved) bipA (or typA) gene for the specific detection of Salmonella spp., including the Salmonella serovars undetected by invA-based PCR methods. ${ }^{[18]}$ Then, the primer pair described for PCR amplification of an 86 bp DNA fragment specific of pathogenic Salmonella was successfully adapted with minor modifications for solid-phase helicase dependent amplification (HDA) by our group ${ }^{[19]}$ Herein we propose the use of this set of primers suited for HDA to gain more insights into the on-solid recombinase polymerase amplification (on-solid RPA).

Unlike PCR, there is no primer design software specific for RPA; however, practical experience along with mechanistic knowledge about the enzymes involved in this isothermal polymerization have allowed to establish a series of recommendations to be kept in mind. ${ }^{[20]}$ Among them, the primer length is, perhaps, one of the most distinctive of RPA. It should be at least 30 nucleotides, though successful RPA amplification has been attained when using PCR primers as well. ${ }^{[15]}$ Another issue of great importance is primer artifacts derived from intra and intermolecular base pairing. They are favored at low temperatures (as those at which RPA operates) as well as by the long size of RPA primers. The feasibility of primer-dimer formation for our set of primers (Tabla S1) was reevaluated at conventional RPA conditions (temperature and ionic strength) using Mfold web server. ${ }^{[21]} A$ Gibbs free energy of $-6.2 \mathrm{kcal} \mathrm{mol}^{-1}$ was predicted for primers hybridization. This value does not completely exclude the unwanted interactions; although, in the presence of recombinase and SSB proteins the probability would be even lower. In consequence, we decided to test this set of primers.

Firstly, RPA amplification was evaluated qualitatively using agarose gel electrophoresis. For this, $3 \times 10^{7}$ genomic units of Salmonella and a negative control without template (negative template control or NTC) were subjected to amplification at 37 ${ }^{\circ} \mathrm{C}$ for $40 \mathrm{~min}$ in the thermal block in the presence of both primers, $480 \mathrm{nM}$ each. After RPA and prior to performing gel electrophoresis, the reaction mix was purified as indicated for PCR products (experimental section) in order to prevent smearing on the gel. The analysis of the purified post-amplified solutions by agarose gel electrophoresis revealed a band corresponding to products of the expected size ( $86 \mathrm{bp})$ in the positive control, while no band was observed in the absence of template (Figure S1). These results indicate that the pair of primers designed for PCR and adapted for HDA amplification is 
also suitable for solution-phase RPA amplification under conventional conditions.

\section{Solid-phase RPA of Salmonella specific ds-DNA}

Next we tested the efficiency of this set of primers for solidphase RPA amplification by using gold chips to build the sensing surface through the formation of a binary thiol-based selfassembled monolayer (SAM), a surface chemistry widely used in electrochemical genosensors. ${ }^{[22]}$ After exposing the cleaned gold working surface to a thiolated primer solution, we selected as a blocking agent the aromatic thiol p-aminothiophenol in place of the traditional 6-mercaptohexanol, because $p$ aminothiophenol results a more beneficial backfilling agent in terms of lower background signal and higher stability of the resultina surfaces. ${ }^{[23]}$

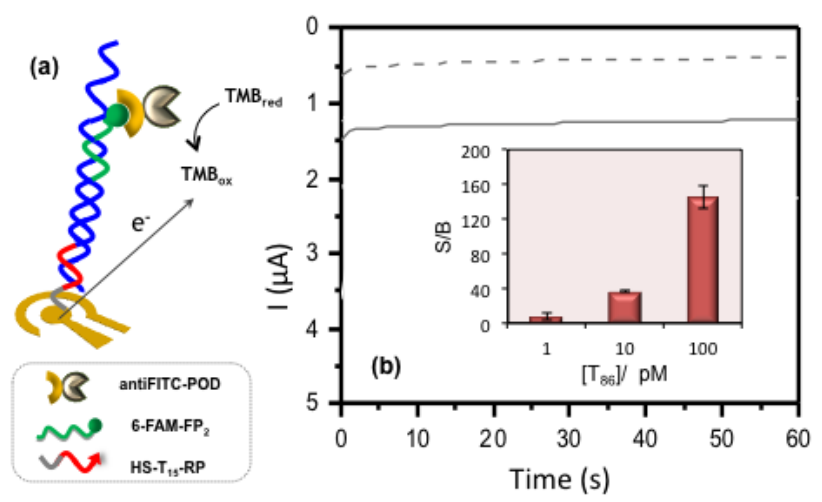

Figure 1. (a) Schematic representation of the enzyme-amplified electrochemical detection of RPA amplicons on gold chips. (b) Biosensor response for 86 bp dsDNA ( $\left.T_{86}\right)$ or Salmonella genome after recombinase polymerase amplification with $\mathrm{HS}-\mathrm{T}_{15}-\mathrm{RP}$ immobilized on the chip surface and $480 \mathrm{nM}$ of $6-\mathrm{FAM}-\mathrm{FP}_{2}$ in solution, at $37^{\circ} \mathrm{C}$ for $40 \mathrm{~min}$. Chronoamperograms corresponding to (dashed line): blank, (solid line):0.17 pM Salmonella genome. Inset: Signal to blank ratio $(S / B)$ recorded for different concentrations of $T_{86}$.

The length of the target DNA is one of the key parameters affecting amplification efficiency; therefore to explore whether the on-surface amplification is possible with the selected pair of primers we started using an 86-bp DNA fragment, $T_{86}$, obtained by PCR from Salmonella genome as the template for the RPA reaction. The amplification was carried out using just the fluorescein-modified forward primer (6-FAM-FP $)$ in solution with the purpose of generating a surface-immobilized amplicon by extension of the reverse primer attached to the gold surface. The amount of 6-FAM-tagged amplicon produced was determined after enzyme labelling with peroxidase conjugated to an anti-FITC Fab fragment (antiFITC-POD) by means of the antiFITC-fluorescein affinity interaction. $P O D$ catalyses the reduction of $\mathrm{H}_{2} \mathrm{O}_{2}$ and the resulting oxidized form of the enzyme oxidizes the co-substrate $\mathrm{TMB}_{\text {red }}$ into $\mathrm{TMB}_{\mathrm{ox}}$, which is detected by chronoamperometry (Figure 1). The plateau current thus recorded is used as the biosensor response. This one was found to increase with the increasing amount of $T_{86}$ in the RPA reaction mixture, and the corresponding signal to blank ratio $(\mathrm{S} / \mathrm{B})$ for each evaluated $\mathrm{T}_{86}$ concentration is shown in Figure 1.
Poor reproducibility was obtaining for $1 \mathrm{pM} \mathrm{T}_{86}$, the lowest assessed concentration, as just one in three replicates gave a specific positive amplification, i.e. a signal significantly different from the background, which could match the minimum amount of target that can be detected. When extending the amplification time from 40 to $60 \mathrm{~min}$, a slight improvement was achieved since 2 in 3 replicates could be clearly distinguished from the blank.

The presence of crowding agents in the RPA medium to emulate intracellular environment ${ }^{[24]}$ gives rise to high viscosity, which hampers the diffusion of reagents through the solution and towards the surface. Moreover, considering the relatively low temperatures at which RPA operates (37-42 $\left.{ }^{\circ} \mathrm{C}\right)$, the mixing effects of thermal convection are reduced with respect to PCR and to some isothermal variants such as LAMP and HDA that occur at around $65^{\circ} \mathrm{C}$. Both issues prompt the slow-down of the transport of target to the surface, and this effect is particularly adverse in samples with low concentration of target sequences. Taking the above elements together, we consider the possibility of including a mixing step to improve the assay performance. ${ }^{25]}$ To do so, we utilized a thermal block with an automatic mixing system by coupling an adapter for PCR plates on which the sensor chip was placed. This way, 10 minutes after the beginning of the reaction, a 10-min mix step was implemented and, during the remaining 20 minutes, incubation was carried out statically. However, we did not observe any improvement of amplification efficiency (data not shown).

As modifications in the reaction time and the deployment of dynamic incubation (movement of the sensing chip) were fruitless for improving reliability of the assay at low target concentrations, we evaluated the influence of incorporating both forward and reverse primers in the RPA solution. As previously reported for $\mathrm{RPA}^{[13-14]}$ and other isothermal amplification techniques ${ }^{[19]}$, small amounts of DNA can be reliably detected as a consequence of an enhanced amplification efficiency when both members of the primer pair are present in the solution. Onsurface RPA amplification of $1 \mathrm{pM} \mathrm{T}_{86}$ as well as the corresponding negative template control were therefore conducted by introducing 6-FAM-FP 2 and RP in solution at 480 $\mathrm{nM}$. As a result, we observed a slight improvement in assay reproducibility, without significantly affecting the signal to blank ratio (data not shown).

\section{Solid-phase RPA of Salmonella genome}

To further demonstrate the usefulness of the proposed assay, we carried out the on-chip amplification of complete bacterial genome, coinciding with the market released of a liquid form of the basic RPA kit. The classical commercial RPA assay (RPA TwistAmp® Basic kit) consists of individual vials with lyophilized reagent pellets as well as reaction buffer and magnesium acetate to activate the enzymes. Although very convenient for routine analysis and its implementation in low resource settings, this configuration greatly reduced the parameters that can be modified to optimize the assay performance. In the new format of the basic RPA kit, the components previously supplied in the freeze-dried pellets are 


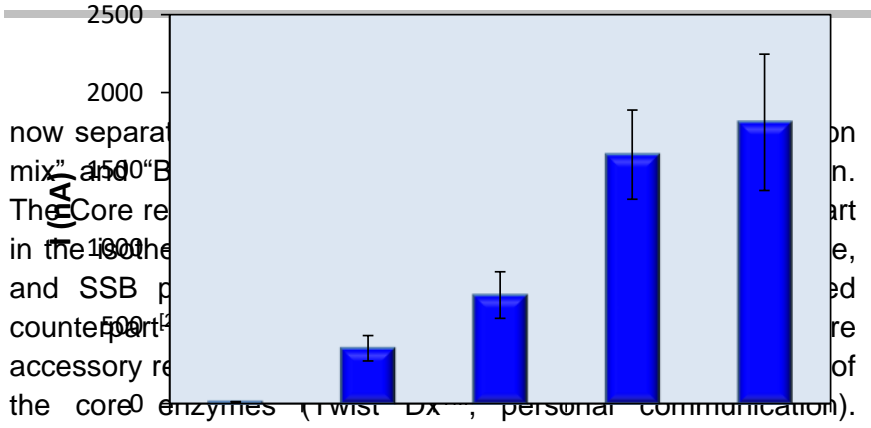

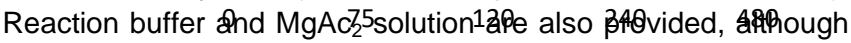
dNTPs are not.

[ 6-FAM-FP] (nM)

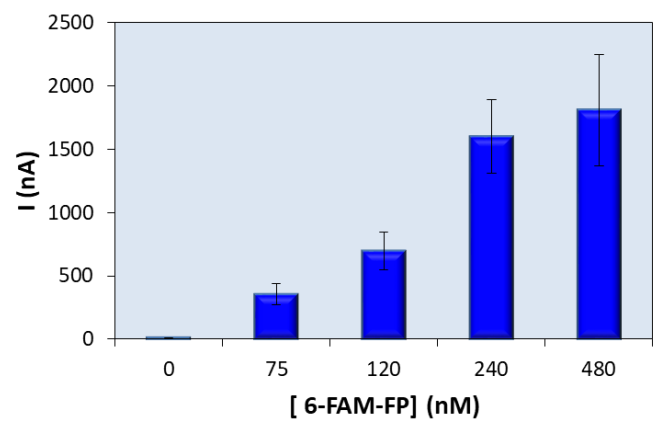

Figure 2. Effect of the in-solution forward primer concentration, 6-FAM-FP, on the electrochemical signal for the negative template control (NTC) assay.

Using the protocol recommended by the manufacturer for this new kit, we incubated Salmonella genome at $37^{\circ} \mathrm{C}$ for 40 min on gold surfaces modified with the binary $\mathrm{HS}-\mathrm{T}_{15}-\mathrm{RP}+p-$ aminothiophenol SAM. Moreover, an analogous experiment without DNA template served as a negative control. Under these conditions, we observed a clear difference in the electrochemical response recorded in the presence of the pathogenic bacterium DNA at a concentration level of $0.17 \mathrm{pM}$, with a signal 2 times larger than that corresponding to the negative control. However, we observed a signal much higher than usual when the assay was performed in the absence of template. In order to investigate the origin of these unexpected results, different 6FAM-FP 2 concentrations ranging from 480 to $75 \mathrm{nM}$ were employed to perform negative control assays. As shown in Figure 2, the electrochemical signal for the negative control decreased as the in-solution forward primer concentration diminished. It seems to indicate that the new kit formulation could somehow promote undesired interactions of 6-FAM-FP with the sensing surface, since the used quantities of RPA reagents may not be exactly the same as those fixed in the freeze-dried pellets. Concomitantly, the S/B ratio increased from 2 to 5 , so according to these experimental results a forward primer concentration of $75 \mathrm{nM}$ was fixed in the RPA amplification solution hereafter.

Furthermore, with the aim of enhancing the efficiency of specific amplification versus nonspecific one, we took advantage of the higher degree of flexibility provided by the liquid format to assess different volumes of the Core reaction mix, and the obtained results are depicted in Figure 3. The increase in Core reaction mix volume led to a decrease in the background signal, which concurs with a more effective stabilization of 6-FAM-FP by recombinase and SSB proteins, thus circumventing the interaction among primers; however, a significant drop-off of specific signal was concomitantly recorded which could be due to a deleterious effect of high polymerase concentration. Nevertheless, the analysis in terms of signal to blank ratio showed very similar values when utilizing the usual volume, 2.5 $\mu \mathrm{L}$, and $50 \%$ more volume; therefore, just as a matter of reagent savings, $2.5 \mu \mathrm{L}$ of Core reaction mix were used hereafter.

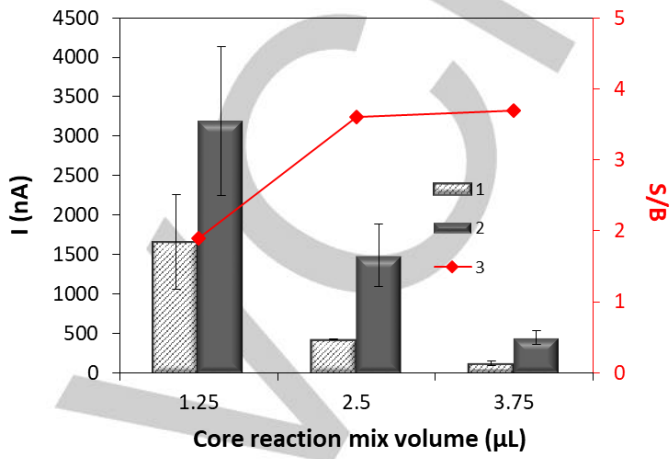

Figure 3. Effect of core enzymes concentration (volume of Core reaction mix) on the signal of the negative control (striped gray), and specific amplification products from $12 \times 10^{6}$ genomic units of Salmonella (solid gray). Signal to blank ratio, $S / B$, is also represented in the secondary $y$-axis. More details in the main text.

Another question that emerges is if solid-phase RPA amplification is actually happening or, on the contrary, it takes place in solution and the resulting products are captured by the sensing monolayer. In this regard, when just the forward nonimmobilized primer is present in the reaction mix, two different mechanisms compatible with the isothermal RPA technology could be envisioned. If the amplification is initiated in solution using solely the forward fluorescein-labelled primer (Figure 4a), only one strand of the Salmonella genome will be amplified resulting in an amplicon longer than 86 base pairs, given that the reverse primer is not present in solution to limit amplicon length (Figure 4a, steps 1-3). Afterwards, the generated 6-FAM labelled amplicons act as the template for on-solid amplification. These are scanned by the recombinase-reverse surface-anchored primer complex, seeking the homologous sequence and favouring the primer insertion in that position (Figure 4a, step 4). The single-stranded DNA binding protein stabilizes the displaced DNA strand and the elongation of the solid-phase attached primer by polymerase leads to the 6-FAM modified 86 bp dsDNA, with a single-stranded tail at the proximity of the electrode surface (Figure 4a, step 5). This latter would be detected electrochemically after a tagging step with the redox enzyme.

Another possibility is a totally interfacial amplification (Figure 4b). In this case, the recombinase facilitates the hybridization between the Salmonella genome and the reverse primer on the solid surface (Figure 4b, step 1) and then the immobilized primer is extended by the DNA polymerase (Figure $4 b$, step 2). As a result, attached label-free amplicons are produced (Figure $4 \mathrm{~b}$, step 3), which serve subsequently as the template for a second surface amplification process involving the 6-FAM modified forward primer present in solution (Figure 4b, steps 4-6). Once 
again, upon enzyme labelling, the fluorescein-labelled immobilized amplicon, presenting in this case ssDNA overhangs arranged towards the solution, would be determined by chronoamperometry.
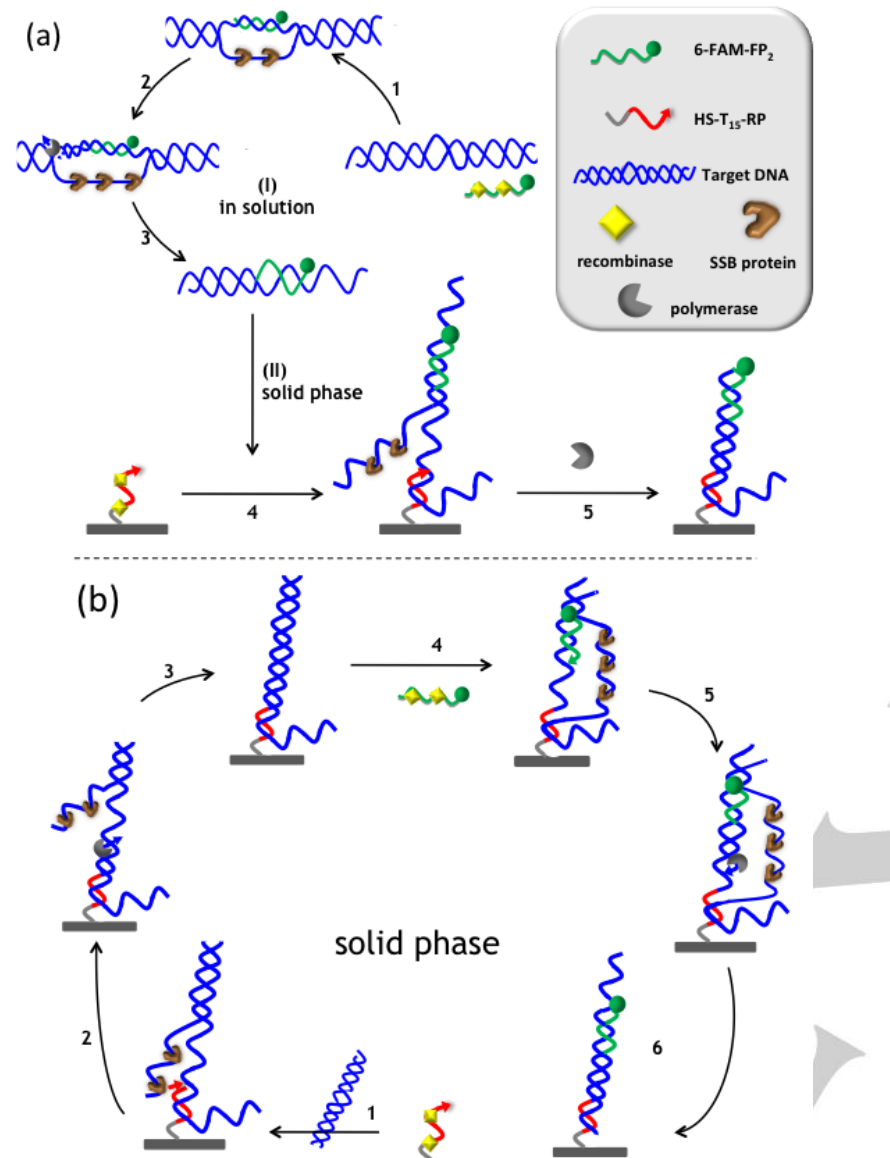

Figure 4. Schematic reaction mechanisms for signal generation during solidphase RPA with the reverse primer attached to the surface and the labelled forward primer in solution: (a) The primer in solution initiates target amplification and it is completed on the surface. (b) The immobilized primer acts as starting point for on-chip RPA amplification.

In order to decipher the amplification mechanism that takes place and to clarify whether on-surface RPA is being effectively produced, further experiments were carried out by using the anchored reverse primer with a dideoxynucleotide at the 3'terminus, $\mathrm{HS}-\mathrm{T}_{15}-\mathrm{RP}^{*}$, to build sensing surfaces. These surfaces were challenged to RPA amplification in the presence of 0.42 $\mathrm{pM}$ Salmonella genomes or $10 \mathrm{pM} \mathrm{T}_{86}$. Likewise, a negative template control was performed in parallel. The lack of the 3'-OH group necessary for the formation of a phosphodiester bond between nucleotides renders this primer unable to be elongated by polymerases; therefore, any recorded signal superior to the background current would be derived from amplicons entrapped somehow by the sensing surface. Using binary monolayers including the blocked-primer no signals higher than the background were obtained (Figure 5). These results ruled out an amplification mechanism starting in solution, and upon entrapment of a 6-FAM-amplicon continued on the surface (Figure 4a). On contrary, they are consistent with an entirely interfacial amplification mechanism like that detailed in Figure 4b, which entails the elongation of the surface-attached primer. This mechanism has also been suggested by Seidel and coworkers $^{[14 \mathrm{~b}]}$, although no experimental evidence was provided. Strikingly, the current responses in the presence of DNA template (Salmonella genome or PCR-generated amplicons) were systematically lower than those for blank experiments. It could be plausibly explained by taking into account the pairing of 6-FAM-FP 2 with the target DNA in solution, which minimizes non-specific interactions between 6-FAM forward primer and the immobilized reverse primer, main responsible for background signals as discussed above.

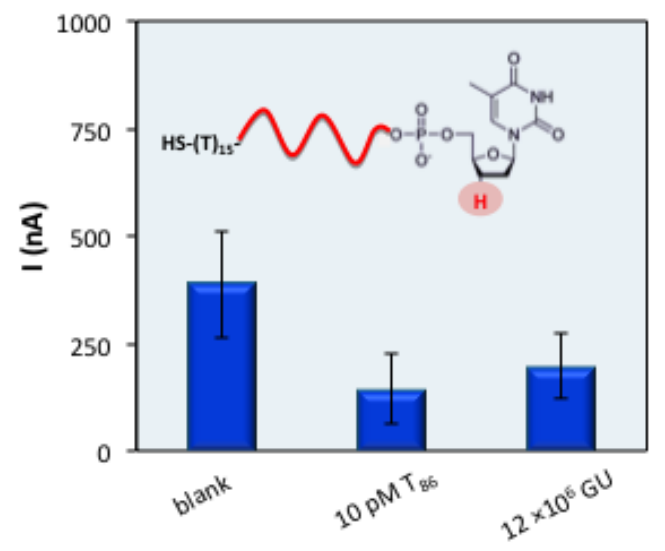

Figure 5. Effect of using a 5'-thiolated reverse primer blocked at its 3'extremity by a dideoxythymidine monophosphate $\left(\mathrm{HS}-\mathrm{T}_{15}-\mathrm{RP}^{\star}\right)$ on the electrochemical response derived from on-surface RPA reaction.

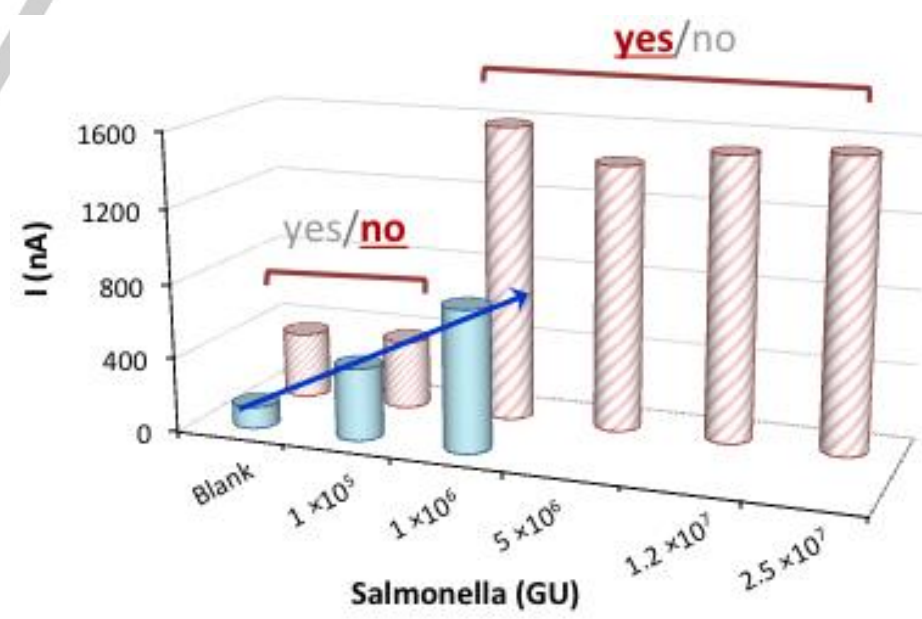

Figure 6. Chronoamperometric signal derived from recombinase polymerase amplification on the gold surface for different starting genomic units of Salmonella at $37^{\circ} \mathrm{C}$, for a RPA reaction time of: (striped red) $40 \mathrm{~min}$, (plain blue) $30 \mathrm{~min}$. 
The on-surface electrochemical assay readily detects $1 \times 10^{6}$ genomic units, i.e. bacterial genomes present in the sample, after $40 \mathrm{~min}$ of amplification. However, we observed a high and very similar current response for increasing amounts of Salmonella DNA (Figure 6), which could point to the saturation of the sensing monolayer (all available immobilized-reverse primers would have been enzymatically extended). Consequently, under these conditions the assay may be used for the binary (yes or no readout) detection of the pathogen, considering as negative those samples giving a signal below the signal corresponding to the mean of the blank plus three times its standard deviation (threshold value).

In order to convert the current yes/no assay in a sensing device capable of discriminating different number of bacterial genomic units, a decrease in the enzymatic amplification time would be useful. Accordingly, we performed the same experiment reducing the reaction time to $30 \mathrm{~min}$. As shown in Figure 6, this reduction of RPA amplification time makes quantitative detection of $10^{5}$ genomic units (GU) possible, with a signal to blank ratio of 3.4 , improving detectability and shortening the total time of the assay. The limit of detection of the assay, defined as the lowest level of the target analyte with at least $95 \%$ of positive replicates ${ }^{[28]}$, is in this case $10^{5} \mathrm{GU}$.

The assay selectivity was also tested by evaluating the response to Legionella pneumophila genome, a gram-negative bacterium that, along with Salmonella, can be found in water samples. In the presence of $10^{6} \mathrm{GU}$ of $L$. pneumophila, the recorded signal was found to be indistinguishable from the negative control $(S / B=1)$, which supports the selectivity of the assay.

\section{Conclusions}

In this work, solid-phase RPA technology on an electrochemical platform has been adapted for the specific detection of Salmonella genome. The strategy involves the immobilization of the reverse primer onto the gold surface of a chip by chemisorption and the incorporation of the fluoresceintagged forward primer in solution for an entirely interfacial isothermal amplification of the target. We have unambiguously demonstrated that on-surface RPA occurs through a direct extension of the attached primers ruling out the possibility of trapping of previously amplified products in solution. After optimization of reaction time and enzymes concentration, $10^{5}$ genomes $\left(2 \times 10^{3}\right.$ copies $\left./ \mu \mathrm{L}\right)$ could be reliably measured even if suboptimal primers, derived from PCR/HDA methods, are employed. Further investigation into the effect of primer design on the background signal, pushing down the minimum amount of genome detected, is required. On account of its exponential nature, primer design simplicity as well as its ability to proceed optimally at cellular temperature, reducing the heating costs in terms of instrumentation and energy supply, RPA is expected to be more suitable for point-of-need analysis than other solidphase isothermal amplification strategies such as Rolling Circle Amplification (RCA) or helicase-dependent amplification (HDA).

\section{Experimental Section}

\section{Instrumentation}

Electrochemical measurements were performed with an Autolab PGSTAT 12 potentiostat/galvanostat controlled by a computer by means of the software package NOVA 2.1 (Eco Chemie B.V., Utrecht, The Netherlands) with a MUX.MULTI4 multiplexor module (Metrohm Autolab B.V., Spain), as well as a connector (DRP-CAC8X, Metrohm-DropSens, Spain). This setup allows eight sequential electrochemical measurements.

The assays were conducted on chips consisting of 16 independent electrochemical cells defined by a polycarbonate mask and distributed in two rows (Figure S2). Each cell consists of a gold working electrode of 2 $\mathrm{mm}$ in diameter, surrounded by a counter electrode of the same metal. As a reference electrode, an $\mathrm{AgIAgClIKCl} \mathrm{(3} \mathrm{M})$ electrode isolated from the test solution by a $\mathrm{KNO}_{3}(3 \mathrm{M})$ salt bridge inside a syringe was used; it was disposed on top of each cell in contact with the solution that contains. All the potentials are referred to this reference electrode and a new modified cell was used for each assay.

\section{Reagents}

All reagents were of analytical grade and they were acquired commercially. p-Aminothiophenol, dithiothreitol (DTT), 3,3',5,5 tetramethylbenzidine (TMB) liquid substrate for peroxidase in a ready-touse reagent format including $\mathrm{H}_{2} \mathrm{O}_{2}$, phosphate buffered saline $10 \times$ concentrate (10x PBS), concentrated saline sodium phosphate-EDTA (20x SSPE) pH 7.4 were obtained from Sigma-Aldrich (Spain). 1\% casein blocking solution in $1 \times$ PBS was received from Thermo Scientific (Spain). Anti-fluorescein-peroxidase Fab fragment (antiFITC-POD) was purchased from Roche diagnostics (Spain). Ultrapure water (resistivity: 18.2 $\mathrm{M} \Omega \cdot \mathrm{cm}$ ) was obtained from a Milli-Q System and used to prepare all aqueous solutions, unless specifically noted otherwise.

RPA TwistAmp® Basic kit and TwistAmp® Liquid Basic kit were purchased from TwistDx Ltd (Cambridge, United Kingdom).

HPLC-purified oligonucleotides were purchased as lyophilized powder from Metabion (Germany) and their sequences are shown in Table S1. The thiolated RPA primer was commercially provided in its oxidized (disulfide) form so it was chemically reduced with DTT and subsequently purified using a Sephadex G25 column (NAP-10, GE Healthcare) as described elsewhere. ${ }^{[26]}$ All DNA oligonucleotides stock solutions were prepared with Milli-Q water and stored at $-20^{\circ} \mathrm{C}$ until use.

Both bacterial genome and PCR-amplified 86 bp DNA sequence ( $\left.T_{86}\right)$ specific of pathogenic Salmonella were employed as the target in this work. Genomic DNA of Salmonella enterica subsp. enterica (CECT 878) was obtained from The Spanish Type Culture Collection (Valencia, Spain) in lyophilized format, reconstituted in Milli-Q water, aliquoted $\left(5 \times 10^{6}\right.$ genomic units per microliter, GU/ $\left.\mu \mathrm{L}\right)$, and stored at $4{ }^{\circ} \mathrm{C}$ until use. From this Salmonella genome, a specific 86 bp DNA fragment was obtained by PCR amplification, using the IMMOLASETM DNA Polymerase kit (Bioline, Spain) and two primers, FP and RP (Table S1), previously reported. ${ }^{[17]}$ The initial composition of the reaction mixture was: $1 \times$ PCR buffer, $2 \mathrm{mM} \mathrm{MgCl}_{2}, 0.25 \mathrm{mM}$ dNTPs, $1 \mu \mathrm{M}$ each primer, $1 \mathrm{U}$ Immolase $^{\mathrm{TM}}$ DNA polymerase, $2.5 \mu \mathrm{L}$ of $10^{6} \mathrm{GU}$ of Salmonella genome and DNasefree water to a total reaction volume of $25 \mu \mathrm{L}$. PCR amplification was carried out using an initial denaturation step at $95^{\circ} \mathrm{C}$ for $10 \mathrm{~min}$, followed by 30 cycles each one consisting of $15 \mathrm{~s}$ at $95^{\circ} \mathrm{C}, 30 \mathrm{~s}$ at $60^{\circ} \mathrm{C}$, and 30 $\mathrm{s}$ at $72^{\circ} \mathrm{C}$. A final extra step at $72^{\circ} \mathrm{C}$ for $7 \mathrm{~min}$ allows the extension of any incomplete product. This protocol was implemented in a thermal cycler (GeneAmp® PCR System 2700 thermocycler (Applied Biosystems, Spain)). The resulting $86 \mathrm{bp}$ amplicon was purified with MinElute PCR purification kit (Qiagen) and quantitated by spectrophotometric analysis 
at $260 \mathrm{~nm}$ using a GENESYS ${ }^{T M}$ 10S UV-vis spectrophotometer (ThermoScientific, Spain)

\section{Protocols}

\section{Sensing surface construction}

Gold chips were washed with ethanol and deionized water, and dried with a nitrogen gas flow. Afterwards, 16 wells with a diameter of $8 \mathrm{~mm}$ each and arranged in two rows were defined by sticking a polycarbonate mask onto the chip. Then each well was covered with $50 \mu \mathrm{L}$ of $0.5 \mathrm{M}$ $\mathrm{H}_{2} \mathrm{SO}_{4}$ and the gold working surface was electrochemically conditioned by applying a voltage of $+1.3 \mathrm{~V}$ vs. $\mathrm{AglAgCllKCl}(3 \mathrm{M}) \mathrm{INNO}_{3}(3 \mathrm{M})$ for $30 \mathrm{~s}$ Subsequently, several potential cycles between 0 and $1.3 \mathrm{~V}$ were performed at $100 \mathrm{mV} / \mathrm{s}$ until a stable cyclic voltammogram was recorded. Lastly, chips were rinsed with deionized water and dried under a stream of nitrogen gas just before the formation of the mixed self-assembled monolayer (mixed SAM).

Mixed SAMs were prepared by covering the clean and conditioned gold working electrode surface $\left(0.03 \mathrm{~cm}^{2}\right.$ geometric area) of each well with a 1 $\mu \mathrm{M}$ solution of the thiolated reverse primer (HS-T $15-\mathrm{RP})$ in $2 \times \mathrm{SSPE}$ buffer $\mathrm{pH} 7.4$, at $4{ }^{\circ} \mathrm{C}$ overnight under water-saturated atmosphere, followed by $50 \mathrm{~min}$ of exposure to $1 \mathrm{mM}$ p-aminothiophenol in $2 \times \mathrm{SSPE}$ buffer $\mathrm{pH} 7.4$ at room temperature. Because of the proximity and identical material of the working and counter electrodes in the chip (see Figure S2), high and poorly reproducible blank signals could be anticipated when trying to confine the solution on the gold working electrode. For that reason, it was decided to cover not only the surface of this electrode but also the whole cell with $50 \mu \mathrm{L}$ of $1 \mathrm{mM} p$ aminothiophenol solution, thus passivating the proximal gold counter electrode. The following steps of the assay were carried out in a volume of $50 \mu \mathrm{L}$ as well. Before solid-phase RPA, each well was thoroughly washed with $2 \times$ SSPE buffer $\mathrm{pH} 7.4$ and dried in $\mathrm{N}_{2}(\mathrm{~g})$ flow.

\section{RPA amplification}

RPA reactions were carried out in a final volume of $50 \mu \mathrm{L}$. The TwistAmp® Basic kit was used according to the manufacturer's instructions. Briefly, each freeze-dried pellet was resuspended in a solution containing $29.5 \mu \mathrm{L}$ rehydration buffer, RPA primers in a final concentration of $480 \mathrm{nM}$ each, the DNA template and DNase-free water up to a total volume of $47.5 \mu \mathrm{L}$. Upon mixing with the pipette, $2.5 \mu \mathrm{L}$ of $280 \mathrm{mM}$ magnesium acetate were added to trigger the amplification. The TwistAmp® Liquid Basic kit provides reagents in liquid format and the combination thereof should be carried out in the following order. First, a primer mix is prepared in a tube by addition (per reaction) of $25 \mu \mathrm{L} 2 \times$ reaction buffer, $9.2 \mu \mathrm{L}$ dNTPs in a final concentration of $450 \mu \mathrm{M}$ each, 5 $\mu \mathrm{L} 10 \times$ Basic E-mix, and $2.4 \mu \mathrm{L}$ of each primer (stock solution of $10 \mu \mathrm{M}$ to get a final concentration of $480 \mathrm{nM}$ ). After agitation in the vortex and a short burst of centrifugation, $2.5 \mu \mathrm{L} 20 \times$ Core reaction mix are incorporated in the tube and spinned briefly. Finally, the reaction mixture is completed with the DNA template and $2.5 \mu \mathrm{L}$ of $280 \mathrm{mM} \mathrm{MgAc}_{2}$.

In both cases, to guarantee the simultaneous start of the amplification reactions, $\mathrm{MgAc}_{2}$ was pipetting into the tube wall, thus avoiding the immediate contact with the solution, and then all tubes were spinning at the same time.

RPA detection on the chip

RPA reaction mixtures prepared in vials as described above were added to the wells of the chip, which were sealed with Parafilm®, thus minimizing the possibility of cross-contamination. Likewise, a preheated metal plate was placed on the film to reduce the evaporation of the solution. The isothermal amplification was then performed in an oven (Heraus VT6025, $\pm 0.1^{\circ} \mathrm{C}$ ) at $37^{\circ} \mathrm{C}$ for $40 \mathrm{~min}$, or in a thermal block (Thermomixer comfort, Eppendorf AG, Hamburg, Germany).

Upon washing with $2 \times$ SSPE buffer, a conditioning step with casein blocking buffer $(0.5 \%(\mathrm{w} / \mathrm{v})$ in $1 \times \mathrm{PBS}, \mathrm{pH} 7.4)$ was performed for $15 \mathrm{~min}$. Afterwards, the enzyme labelling was conducted in $50 \mu \mathrm{L}$ of casein blocking buffer including $0.5 \mathrm{U} / \mathrm{mL}$ of anti-fluorescein-POD conjugate. After $15 \mathrm{~min}$ of incubation time at room temperature and protected from light, the surface was cleaned with $2 \times$ SSPE buffer. The immobilized enzyme activity, directly related to the amplicon generated on the surface, was determined by covering the cell with $50 \mu \mathrm{L}$ of the enzyme substrates solution $\left(\mathrm{TMB}+\mathrm{H}_{2} \mathrm{O}_{2}\right.$ ) for $30 \mathrm{~s}$, followed by chronoamperometric detection at $0 \mathrm{~V}$ for $60 \mathrm{~s}$

\section{Acknowledgements}

This work was financially supported by the Spanish Ministerio de Economía y Competitividad (Project No. CTQ2015-63567-R), and co-financed by FEDER funds.

Keywords: bacterial genome detection • electrochemical biosensing • on-surface DNA amplification $•$ recombinase polymerase $\cdot$ Salmonella

[1] (a) H. Qi, S. Yue, S. Bi, C. Ding, W. Song, Biosens. Bioelectron. 2018, 110, 207-217; (b) Y. Zhao, F. Chen, Q. Li, W. Lihua, F. Chunhai, Chem. Rev. 2015, 115, 12491-12545; (c) L. Yan, J. Zhou, Y. Zheng, A.S. Gamson, B.T. Roembke, S. Nakayama, H.O. Sintim, Mol. Biosyst. 2014, 10, 970-1003.

[2] a) R.K. Daher, G. Stewart, M. Boissinot, M.G. Bergeron, Clin. Chem 2016, 62, 947-958; b) A. James, J. Macdonald, Expert Rev. Mol. Diagn 2015, 15, 1475-1489; c) I. Mariñá-Lobato, C.K. O'Sullivan, Trends Anal. Chem. 2018, 98, 19-35.

[3] Z. Chen, H. Yang, N.P. Pavletich, Nature 2008, 453, 489-494.

[4] O. Piepenburg, C.H. Williams, D.L. Stemple, N.A. Armes, PLoS Biol. 2006, 4, 1115-1121.

[5] O. Piepenburg, C.H. Williams, N.A. Armes, D.L. Stemple, US 7666598 b2, Feb. 23, 2010.

[6] Z. Crannell, A. Castellanos-Gonzalez, G. Nair, R. Mejia, A.C. While, R Richards-Kortum, Anal. Chem. 2016, 88, 1610-1616.

[7] S. Santiago-Felipe, L.A. Tortajada-Genaro, S. Morais, R. Puchades, A. Maquieira, Food Chem. 2015, 174, 509-515.

[8] a) A. de-la-Escosura-Muñiz, L. Baptista-Pires, L. Serrano, L. Altet, O. Francino, A. Sánchez, A. Merkoçi, Small 2016, 12, 205-213; b) H.Y Lau, H. Wu, E. J. H. Wee, M. Trau, Y. Wang, J. R. Botella Sci. Rep. 2017, 7, 38896

[9] (a) M. Amiri, A. Bezaatpour, H. Jafari, R. Boukherroub, S. Szunerits, ACS Sens. 2018,3, 1069-1086. (b) E. Palecek, M. Bartosik, Chem. Rev. 2012, 112, 3427-3481.

[10] O. Mayboroda, I. Katakis, C.K. O'Sullivan, Anal.Biochem. 2018, 545 , 20-30.

[11] J. S. del-Río, I. Magriñà-Lobato, O. Mayboroda I. Katakis, C.K. O'Sullivan, Anal. Bioanal. Chem. 2017, 409, 3261-3269.

[12] S. Santiago-Felipe, L.A. Tortajada-Genaro, R. Puchades, A. Maquieira, Microchim. Acta 2016, 183, 1195-1202.

[13] S. Santiago-Felipe, L.A. Tortajada-Genaro, S. Morais, R. Puchades, A. Maquieira, Sensors and Actuators B: Chemical 2014, 204, 273-281.

[14] a) S. Kersting, V. Rausch, F.F. Bier, M. von Nickisch-Rosenegk, Microchim. Acta 2014, 181, 1715-1723; b) A. Kunze, M. Dilcher, A. Abd El Wahed, F. Hufert, R. Niessner, M. Seidel, Anal.Chem. 2015, 88, 898-905. 
[15] a) Y. Shin, A.P. Perera, K.W. Kim, M.K. Park, Lab Chip 2013, 13, 2106 2114; b) Q. Liu, J. Nam, S. Kim, C.T. Lim, M.K. Park, Y. Shin, Biosens. Bioelectron. 2016, 82, 1-8.

[16] O. Mayboroda, A. Gonzalez-Benito, J.S. del Rio, M. Svobodova, S. Julich, H. Tomaso, C.K. O'Sullivan, I. Katakis, Anal. Bioanal. Chem. 2016, 408, 671-676.

[17] S. Umesha, H.M. Manukumar, Crit. Rev. Food Sci. Nutr. 2018, 58, 84104.

[18] L. Calvó, A. Martínez-Planells, J. Pardos-Bosch, L.J. García-Gil, Food Anal. Methods 2008, 1, 236-242.

[19] S. Barreda-García, R. Miranda-Castro, N. de-los-Santos-Álvarez, A.J. Miranda-Ordieres, M.J. Lobo-Castañón, Chem. Comm. 2017, 53, 9721 9724.

[20] https://www.twistdx.co.uk.

[21] M. Zuker, Nucleic Acid Res. 2003, 31, 3406-3415.

[22] R. Miranda-Castro, N. de-los-Santos-Álvarez, M.J. Lobo-Castañón, Electroanalysis 2018, 7, 1229-1240.
[23] a) S. Moura-Melo, R. Miranda-Castro, N. de-los-Santos-Álvarez, A.J. Miranda-Ordieres, J. Ribeiro Dos Santos Junior, R. da Silva Fonseca M.J. Lobo-Castañón, Anal.Chem. 2015, 87, 8547-8554; b) R. MirandaCastro, R. Sánchez-Salcedo, B. Suárez-Álvarez, N. de-los-SantosÁlvarez, A.J. Miranda-Ordieres, M.J. Lobo-Castañón, Biosens. Bioelectron. 2017, 92, 162-170.

[24] P.E. Lavery and S.C. Kowalczykowski, J.Biol.Chem. 1992, 267, 93079314

[25] L. Lillis, J. Siverson, A. Lee, J. Cantera, M. Parker, O. Piepenburg, D.A. Lehman, D.S. Boyle, Molecular and Cellular Probes 2016, 30, 74-78.

[26] O.W. Stringer, J.M. Andrews, H.L. Greetham, M.S. Forrest, S. Mathew, Nat. Methods 2018, 15, I-III.

[27] R. Miranda-Castro, P. de-los-Santos-Álvarez, M.J. Lobo-Castañón, A.J. Miranda-Ordieres, P. Tuñón-Blanco, Anal. Chem. 2007, 79, 4050-4055

[28] S.A. Bustin, V. Benes, J.A. Garson, J. Hellemans, J. Huggett, M. Kubista, R. Mueller, T. Nolan, M.W. Pfaffl, G.L. Shipley, J. Vandesompele, C.T. Wittwer, Clin. Chem. 2009, 55, 611-622. 


\section{Entry for the Table of Contents}

\section{ARTICLE}

The mechanism for the selective and isothermal amplification with electrochemical detection of Salmonella genome on gold chips is investigated. The recombinasepolymerase reaction takes place directly on the surface, elongating the anchored primer, which enables the detection of $10^{5}$ genome units.

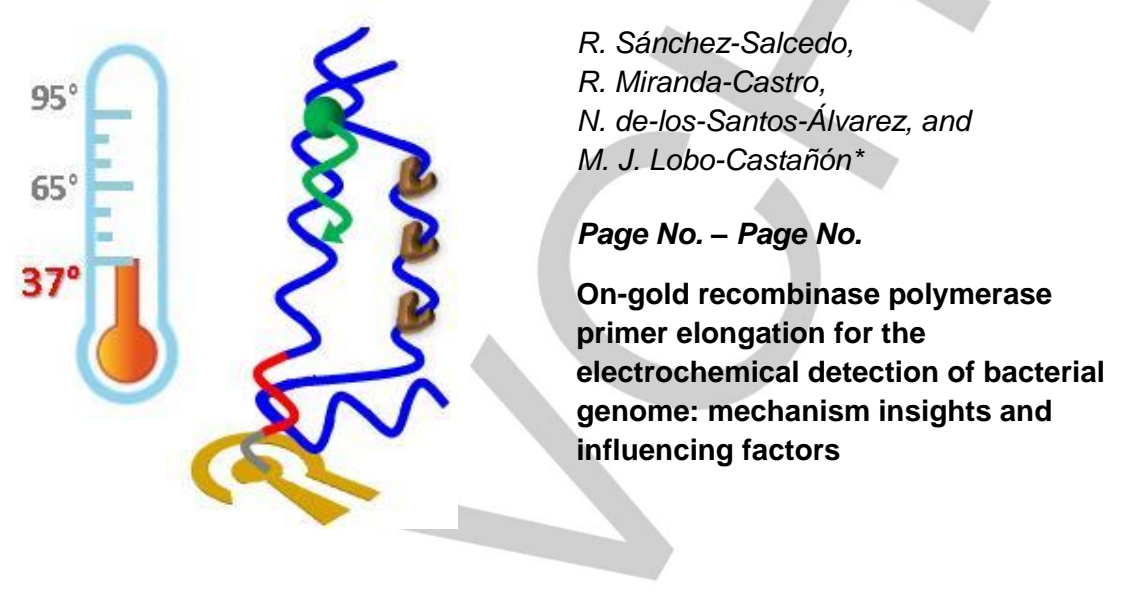

